\title{
LA TEORÍA GENERAL \\ DE LA FORMA DE ROBERT SUMMERS \\ (A propósito de Robert S. Summers, \\ Form and Function in a Legal Sytem. A general Study) *
}

César Arjona **
ESADE

RESUMEN. El propósito de este trabajo es introducir y analizar la teoría general de la forma, del profesor R. SUMmers. Basándose en el concepto de forma (que el autor define como "ordenación sistemática y tendiente a un fin»), SUMMERS presenta una teoría ambiciosa de gran importancia epistemológica. Propone una nueva manera de contemplar el sistema jurídico, la perspectiva de la forma, al tiempo que subraya las insuficiencias de la perspectiva de la regla que caracteriza el pensamiento de iusfilósofos tan importantes como H. KELSEN O H. HART. Así, en el análisis de SUMmERS, las reglas no constituyen el elemento central, como es el caso en el positivismo jurídico tradicional, sino un tipo de unidad jurídica entre otras. Este artículo analiza tanto la dimensión estructural (anatómica) como funcional (fisiológica) de esta nueva teoría. Además, la sitúa dentro del marco de la filosofía jurídica contemporánea, en la que está llamada a ocupar una posición relevante.

Palabras clave: perspectiva de la forma, perspectiva de la regla, epistemología, positivismo jurídico.

ABSTRACT. The purpose of this paper is to introduce and analyse professor R. SUMmERs' general theory of form. Based on the concept of form (defined by the author as a «purposive systematic arrangement»), SUMMERS advances an ambitious theory of epistemological import. He proposes a new way to look at the legal system, the form-oriented approach, while underlying the insufficiencies of the rule oriented-approch that characterises the thought of central legal philosophers such as H. KELSEN and H. HART. Thus, in SUMMERS' analysis, rules are not the dominant element, as it is the case in traditional legal positivism, but just one kind of legal unit among others. The paper analyses both the structural (anatomy) and functional (physiology) dimensions of the new formal theory of law. Furthermore, it puts the theory within the framework of contemporary legal philosophy, in which it is destined to be in a relevant position. tivism.

Keywords: form-oriented approach, rule oriented-approach, epistemology, legal posi-

* R. S. SumMERS, 2006: Form and Function in a Legal System. A general Study, Cambridge: Cambridge University Press.

** Fecha de recepción: 28 de septiembre de 2008. Fecha de aceptación: 30 de octubre de 2008. 


\section{INTRODUCCIÓN}

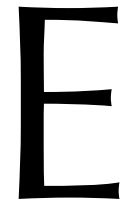

1 propósito de este escrito es presentar uno de los desarrollos más ambiciosos de la filosofía del Derecho de los últimos años: la teoría general de la forma de R. Summers. Para ello tomaré como referencia su obra Form and Function in a Legal System, la cual supone la culminación de más de una década de estudios sobre distintos aspectos formales del Derecho que cabe englobar dentro de esa teoría general ${ }^{1}$. A diferencia de la mayoría de publicaciones iusfilosóficas, que están integradas dentro de alguna tradición teórica bien definida, SuMMERS plantea aquí una teoría general del Derecho propia y original, la cual basa en el elemento que ha dominado su producción académica reciente: el de forma ${ }^{2}$.

«Forma» es un término ambiguo y filosóficamente complejo, que Summers define como purposive systematic arrangement ${ }^{3}$. No se trata de una expresión de traducción sencilla, y adopto la ya empleada por M. ATIENZA: «ordenación sistemática y tendiente a un fin» ${ }^{4}$. El punto de partida de Summers es que el estudio de la forma en el Derecho tiene gran relevancia tanto teórica como práctica, a pesar de lo cual no ha sido afrontado con la debida atención por la teoría jurídica occidental, y en particular por el positivismo jurídico dominante.

Pero el trabajo no sería tan ambicioso como es en realidad si sólo tratase de resaltar un elemento de los sistemas jurídicos que ha pasado tradicionalmente desapercibido. De ser así, la novedad de la teoría se hallaría en la dimensión ontológica; sin embargo, a mi entender, el gran valor de la misma hay que situarlo en el nivel epistemológico. Es decir: su principal importancia no radica tanto en mostrar las formas jurídicas como en proponer una nueva manera de ver el Derecho en su conjunto a partir del estudio de la forma. Se trata de lo que SuMMERS denomina form-oriented approach: una manera de aproximarse al fenómeno jurídico a través de la forma u orientada hacia la forma, la cual resulta especialmente fructífera dado que todo aspecto del Derecho tiene un

\footnotetext{
${ }^{1}$ Summers ha publicado, desde comienzos de los noventa, más de una veintena de artículos sobre la importancia de la forma en el Derecho, que pueden considerarse piezas sueltas de la teoría general que presenta en Form and Function in a Legal System. Algunas de ellas fueron publicadas en lengua española en una recopilación de artículos titulada 2001: La naturaleza formal del Derecho, México: Fontamara, (trad. P. LARRAÑAGA).

2 Cabe reseñar que, más allá de libro mencionado en la cita anterior, la obra de SuMMERS no ha recibido en la literatura jurídica en lengua española un tratamiento global. Sí que existen referencias, aunque no abundan, a algunas de las publicaciones de SuMMERS durante los últimos años. Un buen ejemplo de esto lo ofrece el reciente libro de R. GARCía MANRIQUe, 2007: El valor de la seguridad jurídica, México: Fontamara, a lo largo de cuya segunda parte se analizan las posiciones de SUMMERS como defensor de una concepción formal del rule of law y de su valor moral.

3 Se trata de una definición ad hoc pero no arbitraria, ya que es cercana y compatible con muchos usos del término «forma» tanto en el lenguaje ordinario como en el jurídico. Este es un término que ha sido objeto de múltiples tratamientos en la tradición filosófica occidental, desde PLATÓN hasta nuestros días. La precisa definición de SUMMERS es de gran utilidad para el desarrollo de su teoría, que si no podría verse colapsada por una discusión conceptual, e incluso terminológica, al nivel de la filosofía teorética.

${ }^{4}$ La utilizó en «Presentación» a 2001: La Naturaleza formal del Derecho, México: Fontamara. El análisis terminológico y conceptual mediante el que SuMMERS justifica su definición de «forma» corresponde a la lengua inglesa, y es cierto que algunos de sus usos y connotaciones pueden variar para el hablante en lengua española. Sin embargo, como dice acertadamente el traductor de la obra antes citada, P. LARRAÑAGA, eso no es impedimento para la comprensión de la teoría, y buscar términos alternativos al de «forma» allí donde las connotaciones de su uso por SUMMERS no se ajusten a las españolas provocaría demasiada confusión (pp. 20$21, N . \operatorname{del} T$.)
} 
componente formal, y que por tanto la forma abarca la totalidad del sistema. De ahí que aunque me refiera aquí a su teoría mediante la denominación que el propio autor adopta, a saber, «teoría general de la forma», cabría también identificarla como una «teoría general del Derecho a través de la forma», una expresión esta que refleja de manera fiel la ambición de la propuesta de Summers.

\section{LA PROPUESTA EPISTEMOLÓGICA}

\subsection{Orientación hacia la regla vs. orientación hacia la forma}

Desde un punto de vista epistemológico, el libro está atravesado por un constraste entre la propuesta de SUMMERS, form-oriented approach, y lo que él denomina rule-oriented approach. La perspectiva de la regla representa la manera de aproximarse al fenómeno jurídico dominante en la cultura occidental contemporánea. SUMMERS la identifica explícitamente con la filosofía de los dos más importantes exponentes del positivismo jurídico del siglo XX: H. KELSEN y H. HART ${ }^{5}$.

Tanto HART como KELSEN conciben el Derecho esencialmente como un conjunto de reglas, aunque difieren sobre qué tipos de reglas son esas, sobre cómo se organizan, o sobre otras cuestiones que no restan veracidad a la consideración anterior. De ahí que SUMMERS utilice la expresión rule-oriented approach para referirse a lo que hay de común en la manera de aquéllos de ver el Derecho. Según esa perspectiva, el elemento central del sistema son las reglas (puestas por el Estado, ya que hablamos de filósofos iuspositivistas) que pretenden condicionar la conducta de los individuos que se hallan bajo la jurisdicción de ese sistema.

SUMMERS no cree que esa perspectiva sea errónea en un sentido estricto del término, sino más bien insuficiente. Frente a ella, propone un modo de análisis orientado hacia la forma que considera superior tanto a un nivel especulativo (dado que la forma es una característica esencial de la naturaleza del Derecho, y de todo el Derecho) como práctico (puesto que permite mejorar la manera en la que el Derecho puede lograr sus fines, incluidos los más importantes valores sociales $)^{6}$.

Por «forma» entiende SuMmERS, ya se ha dicho, la ordenación sistemática y tendiente a un fin de una serie de elementos. Esos elementos pueden ser reglas, pero no son sólo reglas. Puestas las gafas del form-oriented approach las reglas dejan de constituir el componente esencial del tejido jurídico, siendo sustituidas en esa posición por lo que SuMMERs llama functional legal units, o unidades jurídicas funcionales. Las reglas son

5 Summers llega incluso a hablar del «Hart-Kelsen» mode of analysis (F\&F 4) para enfatizar lo que en común tienen las teorías de ambos autores. Matiza que el modelo de KELSEN, más que rule-oriented se debería calificar de norm-oriented, aunque ambos enfoques pueden pasar por equivalentes a los efectos aquí relevantes (F\&F 73). Las teorías de HART y de KELSEN, cuya importancia SuMMERS no devalúa sino que reconoce, sirven de constante punto de contraste en su presentación de la perspectiva formal. Incide, eso sí, más en el modelo positivista de HART que en el de KELSEN, quizás no sólo por ser posterior o provenir del mundo anglo-sajón, sino también porque SuMmERS fue discípulo personal de HART.

6 Significativamente, SuMmERS no propone el abandono del modelo de la regla, sino la adopción del formoriented approach como primario y principal, y del rule-oriented approach como secundario y complementario $(\mathrm{F} \& \mathrm{~F} 72-73)$. 
un tipo de unidad jurídica funcional, pero hay muchos otros que no son ni más ni menos importantes que ellas, como las instituciones, las metodologías, y otros. Las functional legal units constituyen así las unidades básicas de análisis del sistema jurídico.

Esta es una innovación importante, y un punto capital en la teoría de Summers. Cuáles son, y cómo se organizan, esas unidades funcionales, es algo de lo que hablo en el apartado 3 de este escrito, e invito a quien desee obtener un panorama general de la teoría a pasar directamente a ese punto. Antes, sin embargo, desarrollaré algo más, aunque sea con un carácter específicamente académico, la propuesta epistemológica de SUMMERS, poniéndola en contacto con otros desarrollos importantes de la filosofía del Derecho contemporánea.

\subsection{En particular: reglas constitutivas $v s$. reglas reforzadoras}

Los exponentes del positivismo contemporáneo no eran ciegos, y evidentemente sabían que en el sistema jurídico hay algo más que reglas promulgadas para ordenar conductas. Centrándonos en particular en la versión de HART, existe un acuerdo en que uno de los principales méritos de la misma radica en haber entrado en el concepto de regla con espíritu analítico para distinguir entre distintos tipos de reglas, habiendo acuñado así la exitosa distinción entre reglas primarias y secundarias. Como es bien sabido, las reglas primarias son aquéllas mediante las que se le exige a los individuos que realicen o dejen de realizar determinadas acciones. Las reglas secundarias, por su parte, son reglas que tratan sobre reglas primarias, ya sean las que permiten la creación, cambio y derogación de reglas primarias (reglas de cambio), las que permiten la resolución de disputas que surjan en relación con las reglas primarias y la ejecución de las decisiones mediante las que aquéllas se resuelven (reglas de adjudicación), y las que permiten identificar qué reglas primarias pertenecen al sistema, dotando así a éste de identidad propia (regla de reconocimiento) ${ }^{7}$.

A las reglas secundarias también se las ha llamado reglas constitutivas, ya que en el modelo de HART ellas «constituyen» aquellos elementos del sistema que son distintos a las reglas. Por ejemplo, ¿qué es un tribunal de justicia? Desde el positivismo habría que responder en términos normativos: es un conjunto de reglas que definen cómo se constituye el órgano, establecen procedimientos para su funcionamiento, etc. Existe en definitiva toda una serie de reglas secundarias que constituyen ese elemento del sistema.

SuMMERS afronta esta cuestión proponiendo un cambio terminológico de trascendencia conceptual: a saber, sugiere que esas reglas no se denominen constitutivas sino reforzadoras (reinforcive). No se trata de negar ni la existencia ni la importancia de esas normas, sino, en coherencia con el tono de esta teoría, de atribuirles un alcance más limitado que el que tradicionalmente se les concede. Se aplicaría aquí la acertada expresión de K. WiNSTON, quien en un comentario a un texto de uno de los grandes maestros de SUMMERS, L. FULLER, señala: «[e]n muchas ocasiones, y las instituciones jurídicas no son una excepción, la descripción formal de un organismo en términos de sus re-

\footnotetext{
7 H. L. A. HarT, 1998: El concepto de Derecho, Buenos Aires: Abeledo Perrot, pp. 113-123.
} 
glas constitutivas es el aspecto menos revelador de su carácter como fenómeno social en desarrollo» ${ }^{8}$.

HART conoce de la existencia de los tribunales, y entiende que son de gran importancia para la estructura y el funcionamiento de un sistema jurídico. Pero los conoce y los entiende «a través» de reglas, las reglas secundarias que los constituyen y que son, dentro de su teoría, el elemento esencial del fenómeno jurídico, su unidad básica. Esa manera de ver las cosas hace que los elementos distintos a las reglas (como los tribunales y muchos otros) se afronten de una manera que Summers califica de «oblicua» $(\mathrm{F} \& \mathrm{~F} 73)^{9}$. No es una perspectiva errónea; es, utilizando la expresión de WinSTON, poco reveladora.

Mediante el form-oriented approach, sin embargo, podremos afrontar esos elementos de una manera más iluminadora, más directa, y situarlos en la posición central del análisis jurídico, junto a las reglas (pero no por debajo de las reglas ni derivados de las reglas), como unidades jurídicas funcionales que son. Las reglas jugarán, sin duda, un papel en relación con esos elementos o unidades, ya sean los tribunales u otros, pero ese papel será de refuerzo (reinforcive rules) y no de constitución (constitutive rules), al menos no si lo que se quiere indicar mediante ese término es que tales elementos e instituciones son sólo y nada más que el agregado de reglas que se refieren a ellos.

\subsection{Las críticas de SUMmers y DwOrKIn al positivismo de HART}

La crítica de SUMMERS al positivismo hartiano, y a su perspectiva orientada hacia la regla, puede llevar a pensar al lector mínimamente familiarizado con la filosofía del Derecho contemporánea que aquélla comparte mucho con la muy conocida crítica de DWORKIN, quien habla específicamente del rule model para referirse al modelo de HART frente al que dirige sus ataques ${ }^{10}$. Esta apreciación sería errónea y conviene advertir frente a ella, aunque sí existe una similitud de partida entre los caminos teóricos recorridos por SUMMERS y DwORKIN. Es oportuno revisar este punto para un mejor entendimiento de la teoría que aquí nos ocupa.

SuMMERS reconoce de manera expresa que existe un punto de conexión entre su posición y la de DwORKIN ( $\& \& F 74$, n. 16). Ese punto consiste en que ambos teóricos critican una visión del Derecho (que identifican con HART) como sistema compuesto sólo o esencialmente por reglas. Ambos consideran que dicha visión es pobre e insuficiente.

A partir de aquí, sin embargo, sus posiciones tienen poco que ver, y creo que es importante entender la diferencia para insistir en las profundas implicaciones de la inno-

${ }^{8}$ K. WinSTON (ed.), 2001: The Principles of Social Order, Oxford: Hart, p. 231 (cursiva añadida). SUMMERS fue biógrafo de Fuller; véase R. S. Summers, 1984: Lon L. Fuller, Standford University Press.

9 SuMmers señala: «el estudio de una unidad jurídica funcional realizado principalmente mediante el análisis de los contenidos de reglas reforzadoras que prescriben determinados aspectos de esa unidad es un estudio de dichos aspectos por vía indirecta que no enfoca de frente la forma global, las características formales constitutivas y sus interrelaciones, y los componentes materiales o de otro tipo de la unidad complementarios a su forma y a sus características formales» (F\&F 79).

${ }_{10}$ R. DwORKIN, 1967: «The Model of Rules», 35, University of Chicago Law Review, 14. 
vación epistemológica propuesta por SUMMERS. Lo que DWORKIN viene a decir es que si miramos bien el Derecho (o con más atención, o con más sutileza, de lo que lo hizo HART), veremos que además de reglas hay otro tipo de preceptos, los principios. Pero lo que SuMMERS viene a decir es que si miramos de otra manera el Derecho, veremos que además de las reglas y de los principios, existe toda una serie de elementos, como las instituciones, o las metodologías, y otras unidades jurídicas funcionales distintas a los preceptos, que pasan desapercibidas para la teoría tradicional; y que existe toda una serie de cuestiones de gran importancia sobre las características formales de esos elementos y de sus interrelaciones que pasan igualmente desapercibidas para la teoría tradicional.

Dicho en otros términos, DWORKIN amplía el campo de los preceptos relevantes (o cuando menos establece una distinción dentro del mismo que HART no había realizado), pero continúa manteniendo el foco de la teoría centrado en los preceptos. SuMMERS va más allá: no sólo es limitado ver en las reglas todos los preceptos (pues hay más) sino que es limitado ver en los preceptos todo el Derecho (pues hay mucho más). Por ello, desplaza el foco de la teoría jurídica desde los preceptos hacia la forma. Así, mientras que la innovación de DwORKIN, en cuanto critica el modelo de las reglas, es esencialmente ontológica, SUMMERS eleva la cuestión al terreno epistemológico ${ }^{11}$.

\subsection{Summers y los instrumentalistas: forma vs. formalismo}

En la crítica emprendida por SUMMERS contra la perspectiva de análisis orientada hacia la regla, o rule-oriented approach, resuena también el eco de la tradición instrumentalista americana en filosofía y en Derecho, que durante el siglo Xx dio lugar, entre otras, a la llamada escuela del realismo jurídico ${ }^{12}$. En su búsqueda de la esencia del fenómeno jurídico, los realistas moderaron el valor de la regla, y en su versión más extrema llegaron a desconfiar de él, adoptando lo que se ha denominado «escepticismo sobre la regla» (rule skepticism) ${ }^{13}$. Los realistas se inspiraron en una famosa frase del juez Oliver W. Holmes, «las proposiciones generales no deciden casos concretos» ${ }^{14}$, para sostener que las reglas no son el (único) elemento que determina las decisiones judiciales (y por tanto, según su concepción, el contenido del Derecho), sino que juegan ahí otros factores, muchos de los cuales se habían considerado tradicionalmente ajenos al estudio jurídico.

${ }^{11}$ Me estoy refiriendo, en este epígrafe, al punto específico de la teoría de DwORKIN en que introduce los principios (y las policies) como elementos normativos distintos a las reglas. Evidentemente, la filosofía jurídica de DwORKIN es mucho más amplia y compleja, y contiene críticas al positivismo aún más ambiciosas, también desde un punto de vista epistemológico. No encuentro en ellas parecido alguno con la teoría de SuMMERS y quedan, por tanto, fuera del alcance de este escrito.

${ }_{12}$ Summers es autor de una de las principales monografías sobre el instrumentalismo americano: 1982: Instrumentalism and American Legal Theory, New York: Cornell University Press, Ithaca. A pesar de tratarse ese de un estudio crítico, las posiciones propias de SUMMERS no son totalmente ajenas a la influencia del instrumentalismo.

${ }_{13}$ Estoy utilizando aquí «instrumentalismo» y «realismo» como sinónimos. Esto es una imprecisión, según la obra de SuMMERS citada en la nota anterior, y queda aquí justificada por el alcance limitado de este escrito. También me permito la licencia de caracterizar en pocas líneas el realismo tomando como referencia algunas versiones del mismo, y no otras.

${ }^{14}$ La frase proviene de uno de los más famosos votos discrepantes de Holmes en la Corte Suprema de los Estados Unidos: Lochner vs. New York, 198 U.S. 45 (1905). 
Parece claro que la idea inicial de SummERS, limitar y situar en su debido contexto el papel de la regla como elemento de análisis del sistema jurídico, es compatible con los postulados instrumentalistas y comparte bastante de su espíritu. Sin embargo, el desarrollo que hicieron los realistas difiere radicalmente del propuesto por SuMMERS, hasta el punto de que este atribuye a aquéllos parte de la responsabilidad por el desprestigio que sufre la forma en la cultura jurídica occidental. En efecto, entre las causas que han dado lugar a ese desprestigio, hay que considerar destacadamente la impaciencia hacia la forma que SUMMERS atribuye explícitamente a los realistas, y en particular al primer LLEWELLYN, y que tiene su continuación en movimientos posteriores herederos del realismo, como el análisis económico del Derecho (POSNER) o los critical legal studies (KENNEDY) (F\&F 29) ${ }^{15}$.

Esta consideración peyorativa de la forma, a la que se ve como algo prescindible y molesto que dificulta llegar al núcleo de las cosas, se explica cómo una reacción contra la actitud formalista que dominó la cultura jurídica norteamericana durante el siglo XIX y parte del xx. Me refiero a la que R. PounD denominó «jurisprudencia mecánica», según la cual el razonamiento jurídico debe ser un proceso de pura deduccion lógico formal, aislado en la medida de lo posible del entorno social, a partir de premisas inmutables que deben conservarse a toda costa ${ }^{16}$.

Pues bien, frente a ese telón de fondo, SuMMERS reconoce que «muchas declaraciones contra la forma del primer LLEWELLYN fueron reacciones muy convenientes frente a las metodologías formalistas de interpretación y aplicación de algunos jueces americanos de finales del diecinueve y comienzos del veinte» (F\&F 28). Sin embargo, «al equiparar la forma con lo formalista, esos teóricos (realistas y sus herederos) a veces parecen incluso asumir que podríamos prescindir por completo de la forma en las unidades jurídicas funcionales y aun así tener un sistema jurídico viable» (F\&F 29). Dicho en pocas palabras, los realistas atacan la forma allí donde debían limitarse a atacar el formalismo, y en ese sentido, su posición es difícilmente compatible con la del análisis del Derecho a través de la forma.

En contraste con eso, es significativa aquí la referencia a uno de los juristas más admirados por SuMMERS, R. vON IHERING, quien en la cultura jurídica germana jugó un papel parejo al de los instrumentalistas en Estados Unidos, en tanto en cuanto criticó la escuela de pensamiento dominante en sus días (en el caso, la Jurisprudencia de Conceptos) también por considerarla inapropiadamente formalista ${ }^{17}$. Sin embargo, sus crí-

${ }^{15}$ Por cierto, la especulación sobre las posibles causas que han llevado al desprecio de la forma en la cultura jurídica es un punto importante para la coherencia teórica del sistema de SUMMERS. Dado que este autor ve en la forma un elemento de extraordinaria fecundidad para el análisis jurídico, pero al tiempo asevera que ha sido rotundamente olvidado por los juristas, es necesario plantearse por qué se ha producido ese olvido. Si no existiera una respuesta a dicha pregunta todo su modelo de análisis quedaría teñido por una sospecha. Por ello, el autor americano dedica una sección de su libro a hablar sobre el tradicional rechazo hacia la forma y a sugerir varias explicaciones posibles $(\mathrm{F} \& \mathrm{~F} 24-33)$.

${ }_{16}$ R. POunD, 1908: «Mechanical Jurisprudence», 8 Columbia Law Review, 605. Summers describió extensamente ese método jurídico formalista en 1982: Instrumentalism and American Legal Theory, New York: Cornell University Press, Ithaca, pp. 137-56.

${ }^{17}$ En un irónico escrito, «En el cielo de los conceptos jurídicos», IHERING se recrea en la descripción de ese formalismo, llevando ese estilo de razonamiento a extremos desesperantes hasta el punto de divertir al lector. SumMERs hace referencia expresa a la obra. Existe traducción al castellano en R. VON IHERING, 1987: Bromas y veras en la ciencia jurídica, Madrid: Civitas. 
ticas no se acompañaron de un desprecio por la forma, sino, justo al contrario, de un respeto hacia la forma y del reconocimiento de su importante papel en el sistema jurídico, reconocimiento en parte derivado de su profundo dominio del Derecho romano ${ }^{18}$. Hasta tal punto es así, que SuMMERS admite explícitamente haber encontrado en IHERING la principal inspiración para la elaboración de su teoría general de la forma $(\mathrm{F} \& \mathrm{~F} 36)$.

O sea, se puede honrar la forma aun criticando el formalismo. Pero eso no es lo que hicieron los realistas americanos, ni los practicantes de sus múltiples derivaciones académicas y prácticas. La crítica del formalismo se convirtió generalizadamente en un desprecio por todo lo formal. A partir de ahí, los realistas propusieron sustituir el protagonismo de las denostadas reglas y del razonamiento lógico-deductivo por la utilización de métodos empíricos, provenientes particularmente de la psicología y de otras ciencias sociales. Esto lo identifica SUMMERS con una tercera aproximación al fenómeno jurídico, contraria, como la suya, al rule-oriented, pero muy diferente de su form-oriented, que sería el behavior-oriented approach, o perspectiva behaviorista, según la cual para conocer el Derecho lo más importante es el estudio psicosocial de la conducta de los agentes jurídicos. Y esto es algo completamente alejado de la propuesta de SuMMERS, quien ni siquiera se ocupa de analizar esa alternativa epistemológica dándola por desacreditada (F\&F 72). Repetidamente SuMMERS descarta el recurso a métodos empíricos de investigación, no por considerarlos menores o poco importantes, sino innecesarios para su desarrollo de una teoría general de la forma, la cual se mueve en un terreno esencialmente conceptual ${ }^{19}$.

A pesar de todo lo anterior, sí se entrevé una importante influencia del instrumentalismo americano en la teoría de SUMMERS, pero esto en referencia a otro punto bien distinto, a saber, a la concepción dinámica y funcional del Derecho mediante la que se destaca, tanto o más que su estructura, su carácter de actividad para la consecución de determinados propósitos sociales. De esta tendencia me ocupo más abajo, en el epígrafe 3.2 .

\section{EL SISTEMA DE ANÁLISIS}

\subsection{Dimensión anatómica}

\subsubsection{Las unidades jurídicas funcionales}

Contemplado a través del form-oriented approach, la perspectiva que se obtiene del mapa del sistema jurídico varía sensiblemente de la que nos ofrece el positivismo tradi-

${ }^{18}$ Ese dominio, y cómo generó en IHERING un gran respeto por las formas jurídicas, se puede comprobar en una obra del jurista alemán que Summers cita repetidamente en su libro: Geist des Römischen Rechts (edición en lengua española en 4 tomos, 1904: El espiritu del Derecho romano en las diversas fases de su desarrollo, Madrid: Editorial de Carlos Bailly-Bailliere e hijos). Estoy obviando, por mor de la simplicidad, que en IHERING se dan dos etapas académicas diferentes: una primera, donde es cercano a la escuela de la Jurisprudencia de Conceptos, y una segunda, de carácter pragmático e instrumentalista, en la que critica a aquélla.

${ }_{19}$ En F\&F 94-95 se muestra un importante esfuerzo metodológico para identificar qué tipo de cosas importantes se pueden decir sobre la forma sin necesidad de embarcarse en estudios empíricos. 
cional, centrado éste sobre todo en las reglas. Como se dijo antes, los elementos básicos constitutivos del sistema son, para SuMMERS, las unidades jurídicas funcionales, entre las que se encuentran las reglas pero que no se limitan a éstas.

SuMMERS diseña una estructura para las unidades jurídicas funcionales, divididas en cinco tipos de unidades dentro de cada uno de los cuales se encuentran varios subtipos, según la siguiente clasificación ${ }^{20}$ :

\begin{tabular}{|l|l|}
\hline \multicolumn{1}{|c|}{ Tipos de unidades funcionales } & \multicolumn{1}{|c|}{ Subtipos dentro de cada tipo ${ }^{21}$} \\
\hline Institucionales & Instituciones legislativas \\
& Tribunales \\
& Agencias administrativas \\
& Sociedades y otras entidades privadas \\
\hline Preceptivas (preceptual) & Reglas \\
& Principios \\
& Máximas \\
& Órdenes generales \\
\hline No preceptivas (non preceptual) & Contratos \\
& Ciertos intereses sobre la propiedad \\
& (property interests) \\
& Testamentos \\
\hline Metodológicas & Interpretativas \\
& De redacción (drafting) \\
& Probáticas (fact-finding) \\
\hline Ejecutivas & Sanciones \\
(enforcive or implementive) & Remedios \\
& Otras \\
\hline
\end{tabular}

\subsubsection{La estructura del análisis formal}

Si se observan con atención, se verá que todos los subtipos de unidades jurídicas funcionales se hallan relacionados de alguna manera importante con reglas. Así, por ejemplo, en un sistema jurídico mínimamente desarrollado las sanciones están contempladas en reglas, hay un cuerpo de reglas que establece la estructura y el funcionamiento de los tribunales, etcétera.

Sin embargo, la propuesta de SUMMERS consiste en ir más allá del contenido reglado de las unidades (eso ya lo estudia el positivismo tradicional), aunque sin necesidad

${ }^{20}$ Tomo aquí como referencia la clasificación que realiza SuMMERS en F\&F 54, si bien señala el autor que la enumeración no es exhaustiva. Es decir, pueden existir más unidades funcionales. En ese sentido produce cierta confusión el que en algún momento mencione otras unidades funcionales que no aparecen en ese listado inicial, sin que quede claro a cuál de los tipos anteriores corresponden, como es el caso de la institución de la familia o de la profesión jurídica, a las que se refiere en F\&F 306.

${ }^{21}$ Estos subtipos pueden a su vez dividirse en más. Así, por ejemplo, las unidades funcionales interpretativas pueden referirse a métodos para interpretar contratos, leyes, o constituciones, con características diversas en cada uno de los casos. 
de emprender una investigación empírica al estilo de los enfoques behavioristas propios del instrumentalismo. Para ello diseña una estructura de análisis formal que se puede aplicar a cualquier unidad funcional y que se compone de cuatro elementos:

i) El propósito, esto es, el fin para el que la unidad ha sido diseñada dentro del funcionamiento global del sistema. Recuérdese que la forma se define como una organización tendiente a un fin, con lo que se abre la puerta al estudio fisiológico del sistema del que me ocuparé en el epígrafe posterior.

ii) La forma global, u overall form, que es la que corresponde stricto sensu con la ordenación sistemática y tendiente a un fin de todos los elementos que componen una unidad determinada.

iii) Las características formales, o formal features, de cada una de las unidades. Cuáles sean estas características es algo que puede variar enormemente de unidad a unidad en función de sus propósitos y forma global.

iv) Elementos materiales y otros componentes, la consideración de los cuales es uno de los puntos más originales de la teoría de SUMMERS, ya que éstos suelen quedar radicalmente excluidos de cualquier teoría del Derecho al uso.

\subsubsection{Un ejemplo: la institución legislativa}

El método posee un grado de abstracción considerable, y un ejemplo puede ayudar a entender los distintos estadios de análisis. Tomaré una de las unidades funcionales que SUMMERS analiza en detalle en su obra: una institución legislativa (F\&F 91-135).

Partiendo del paradigma de un órgano legislativo central de cualquier sistema jurídico occidental contemporáneo, SUMMERS afirma que los propósitos que dicha unidad cumple incluyen la representación democrática, la adopción de legislación, la provisión de un foro para el debate público de los principales asuntos de actualidad, el control de la adminsitración (incluido el gobierno), la participación en la política exterior, y el tratamiento de cuestiones financieras.

Para lograr dichos propósitos la institución legislativa posee la forma global de un cuerpo oficial debidamente constituido, con una jurisdicción que abarca todo el sistema, de conformidad con estructuras y procedimientos reconocidos no sólo para crear democráticamente Derecho escrito válido sino también para servir otros propósitos importantes, según han sido enumerados anteriormente.

Esa forma global se compone de distintas características formales de tipo composicional (compositional), jurisdiccional, estructural, procedimental y preceptivo (preceptual). Dentro de cada uno de estos tipos se deberán realizar elecciones formales que dotarán de identidad propia a cada unidad funcional, en este caso, al órgano en concreto que se esté analizando: de su elección y correcta realización dependerá en buena parte el éxito de la unidad funcional.

Finalmente, la unidad se caracterizará también por una serie de componentes materiales que estarán parcialmente determinados por las elecciones de forma que se hayan realizado antes, como pueden ser las instalaciones donde se reúne el cuerpo legislativo, los individuos miembros del mismo, los medios tecnológicos que se utilizan para su funcionamiento, etcétera. 
Como decía, las reglas no desaparecen del panorama sino que se hallan detrás de muchas de esas elecciones de forma, apoyándolas de diversas maneras (de ahí que SuMMERS prefiera hablar de normas que refuerzan, en vez de normas que constituyen). Sin embargo, lo que más importa no es lo que hay detrás sino lo que hay delante, y eso es «forma debidamente diseñada para servir propósitos relevantes». La prevalencia de la forma de una institución sobre las reglas que consolidan dicha forma viene refrendada para SUMMERS por el hecho de que aquellos que se dispongan a crear una institución determinada no pueden ni siquiera empezar a redactar dichas reglas sin antes haber concebido y acordado los propósitos de la institución, su forma global, sus características formales, y los componentes complementarios materiales propios de la institución $(\mathrm{F} \& \mathrm{~F} 95)^{22}$.

\subsection{Dimensión fisiológica}

\subsubsection{El Derecho como actividad}

Uno de los principales méritos del form-oriented approach como método de análisis es su dimensión funcional, la cual permite contemplar el Derecho como algo dinámico, como una herramienta destinada a la consecución de ciertos fines, como una actividad o empresa ${ }^{23}$. Esto, que es algo en general propio del pensamiento jurídico americano, y que entronca con la tradición instrumentalista antes comentada y también con el pensamiento de L. FULLER, se percibe en todos los aspectos de la teoría de SumMERS. Resulta sencillo ofrecer ejemplos de esta dimensión fisiológica: la forma se define como un ordenamiento sistemático tendiente a un fin; en coherencia con ello el primer paso del análisis formal consiste en la determinación de los propósitos servidos por las unidades; éstas se denominan unidades jurídicas funcionales; y la palabra «función» aparece en pie de igualdad con «forma» en el título del libro.

A la hora de captar la dimensión dinámica del fenómeno jurídico, la propuesta epistemológica de SuMMERS es superior a la tradicional visión del Derecho como conjunto de reglas. Claro está que, como siempre, hablamos aquí de una cuestión de énfasis, y no sería en absoluto acertado afirmar que los sistemas positivistas de KELSEN o HART ofrecen una visión puramente estática del Derecho.

Así, el lector de El concepto de Derecho (dejaré aquí de lado a KeLSEN, pues es a HART a quien SUMMERS dedica mayor atención), puede encontrar en esa obra las raíces de una visión funcional del sistema jurídico. Las reglas secundarias, constitutivas, o rein-

${ }^{22}$ De hecho, las reglas son en sí mismas unidades jurídicas funcionales, dotadas de propósito, forma global, características formales y componentes materiales, y al estudio formal de las mismas dedica SUMMERS dos capítulos enteros de su libro.

${ }^{23}$ En el comentario de ATIENZA en el que se analizaba la teoría de la forma aun antes de la publicación de este libro, ya se establecía una conexión esencial entre la concepción de la forma de SUMMERS y la concepción del Derecho como actividad: «si — como... ha escrito SuMMERS - la clave para comprender la ciencia del Derecho radica en la noción de forma, la clave para comprender la noción de forma de SUMMERS — añado yoradica en darse cuenta de que a lo que él se está refiriendo es a la forma -o a las formas- de una actividad»; o, «el significado de fondo de su análisis del Derecho orientado hacia la forma (form-oriented en lugar de ruleoriented) sólo se comprende a partir de su concepción del Derecho como una actividad», ambos en M. ATIENZA, 2001: «Presentación» a La naturaleza formal del Derecho, México: Fontamara, pp. 13-14. 
forcive según SUMMERS, que vienen a suplir las deficiencias que existirían en un sistema primitivo y estático, permiten entender el Derecho como una actividad dinámica, cambiante, adaptada al paso del tiempo y a las necesidades sociales. Además, algunas de sus críticas al positivismo anterior (el modelo del mandato de AUSTIN) contienen precisamente un fuerte componente dinámico, como las que se refieren al problema de la sucesión del soberano y a la persistencia de las normas jurídicas durante el reinado de distintos soberanos ${ }^{24}$.

A pesar de ello, está claro que no considera HART el elemento funcional con la misma centralidad con la que lo hace Summers. Puede que la idea del Derecho como instrumento de ingeniería social no sea en sí misma incompatible con las concepciones hartiana y kelseniana, pero sí desplaza el foco de análisis hacia una dimensión del fenómeno jurídico que en éstas pasa, si no desapercibida, al menos no muy destacada. Esto es lo que apunta SuMMERS al afirmar que «en una perspectiva orientada hacia la regla, existe a menudo la tentación de concebir una unidad jurídica funcional como estática en vez de dinámica. Una perspectiva orientada hacia la forma, sin embargo, concibe dicha unidad combinada, integrada y coordinada con otras unidades dentro de una técnica operativa (operational technique) para la realización de actividades de creación y de aplicación del Derecho» (F\&F 87).

\subsubsection{Los instrumentos de segundo nivel}

En la anterior cita se introduce un nuevo elemento de la teoría de SUMMERS, hasta ahora obviado en esta presentación, pero que tiene capital importancia para entender su adecuación al análisis dinámico. El modelo estructural de tipos y subtipos de unidades jurídicas debe complementarse con los que SuMmERs llama instrumentos sistematizadores de segundo nivel, o second level systematizing devices (entre ellos, las técnicas operativas mencionadas en la cita). Así pues, las unidades jurídicas funcionales que antes veíamos pasan a situarse en un primer nivel y son organizadas por estos instrumentos de segundo nivel para constituir la forma global del sistema jurídico en su totalidad (F\&F 305).

Igual que en el caso de las unidades jurídicas funcionales, SuMMERS ofrece un listado de estos instrumentos de segundo nivel, que son los siguientes ${ }^{25}$ :

- Un orden jerárquico centralizado en una autoridad última dentro de cada esfera jurisdiccional (legislativa, judicial, administrativa, ordenación privada).

- Relaciones entre esas distintas esferas jurisdiccionales, estableciendo criterios de prioridad y de resolución de conflictos.

- Debida unificación de leyes en sectores concretos del ordenamiento (codificaciones, consolidaciones, etc.).

- Uniformización de metodologías (interpretación, redacción, etc.).

- Legitimación y coordinación de instituciones (judiciales y otras) para imponer y aplicar sanciones y otras medidas similares.

${ }^{24}$ H. L. A. HART, 19989: El concepto de Derecho, Buenos Aires: Abeledo Perrot, pp. 64-82.

${ }^{25} \mathrm{E}$ igual que sucedía con las unidades jurídicas funcionales, cabe deducir que esa lista de instrumentos no es exhaustiva, ya que el propio SUMMERS los presenta como los «principales» a ser considerados (F\&F 308). 
- Técnicas operativas sistemáticas que permiten la combinación, integración y coordinación de las unidades jurídicas funcionales de primer nivel.

- Operación sistemática de todas las técnicas anteriores según los principios del Estado de Derecho (rule of law).

Se trata de distintos instrumentos de muy amplio alcance que operan sobre las unidades de primer nivel para dotar al sistema jurídico en su totalidad de una forma glo$\mathrm{bal}^{26}$. De entre todos ellos me voy a detener un instante en uno de los puntos, el de las técnicas operativas, lo cual ayudará a entender el rol que juegan estos instrumentos de segundo nivel en la teoría de SUMMERS, y en particular cómo abren la puerta al análisis dinámico o fisiológico del fenómeno jurídico.

\subsubsection{Las técnicas operativas y el análisis de progresión linear}

«Ninguna unidad funcional específica se emplea sola y de manera independiente», afirma SuMmERS. Al contrario, cualquiera que ésta sea, «dicha unidad se combina racionalmente con otras unidades de primer nivel dentro de una o más de las cinco formas globales de técnicas operativas» (F\&F 327). A esas cinco técnicas SummERs las denomina: i) penal, ii) de reparación de daños (grievance-remedial), iii) administrativa-reguladora, iv) de concesión de beneficios públicos (public benefit conferring), y v) de acuerdo privado (private arranging). Dentro de ellas, o de alguna variación fácilmente reconocible de alguna de ellas, se sitúa todo uso del Derecho en un sistema jurídico occidental contemporáneo.

No sería correcto ver en estas técnicas cajones donde almacenar las unidades funcionales de primer nivel; más adecuado resulta hablar de avenidas por las que circulan dichas unidades, de manera debidamente ordenada. «Las diversas unidades jurídicas funcionales, y por tanto las actividades de legisladores, funcionarios administrativos, ciudadanos, jueces, y otros, están organizadas para operar dentro de estas técnicas, empleadas en progresión linear desde la creación inicial del Derecho hasta su aplicación última. Las unidades específicas, las cuales operan dentro de estas técnicas, son necesariamente dinámicas» (F\&F 327). El Derecho logra sus propósitos, pues, beneficiándose de las sinergias que se producen mediante el funcionamiento de las unidades jurídicas cuando éstas están sistemáticamente ordenadas dentro de las técnicas operativas.

El uso de estas técnicas, y su carácter esencialmente dinámico, viene ilustrado sobradamente en el último capítulo de la obra, en el que el autor se embarca en lo que él denomina un análisis de progresión linear. Éste contrasta con la exposición estructural de las unidades funcionales de primer nivel o de los instrumentos sistematizadores de segundo nivel que ha quedado reflejada en la tabla y la lista anteriores. En el análisis de

${ }^{26}$ Como Summers aprecia, en las teorías de KELSEN y de HART existían instrumentos de este tipo, aunque ellos no los concibieran como tales. Así, la norma fundamental de KELSEN o la regla de reconocimiento de HART podrían considerarse, desde la perspectiva de la teoría que aquí se presenta, instrumentos sistematizadores de segundo nivel. Pero su alcance, aunque trascendental, es limitado. Igual que de entre todas las unidades funcionales estos filósofos se ocuparon centralmente de las reglas, sus conceptos de segundo nivel (norma fundamental y regla de reconocimiento) se refieren precisamente a la sistematización de reglas, y nada más que a eso. Dejan fuera de su campo de acción, pues, mucho de lo que SuMMERS pretende iluminar. 
progresión linear la fisiología cobra todo el protagonismo, y se ve en funcionamiento el sofisticado sistema de múltiples piezas que componen la teoría de Summers.

En particular, el capítulo analiza cómo en el Estado de Nueva York se logró utilizar el sistema jurídico para lograr un propósito de salud pública: la erradicación de la enfermedad del cólera. Es un ejemplo complejo, que permite poner en juego una variedad de elementos de primer y de segundo orden, combinados en distintas técnicas operativas que juegan todas para lograr un mismo fin. SuMMERS aplica los conceptos introducidos durante la obra a la exhaustiva documentación que presenta sobre el caso. El camino de esa historia jurídica está repleto de elecciones sobre la forma en relación tanto con las unidades de primer nivel como con los instrumentos de segundo nivel, incluyendo las técnicas operativas; elecciones estas que cuando fueron apropiadas permitieron el éxito total en el uso del Derecho, en el caso la erradicación de la enfermedad.

\section{UNA ANALOGÍA PARA CONCLUIR}

Pongamos por caso que un lector de la teoría de SumMERS decide, en un arranque de motivación, ir a visitarlo personalmente para discutir con él algunos puntos. Si se presenta sin previo aviso en su casa de la pequeña población de Ithaca, donde se encuentra la Universidad de Cornell, puede correr el riesgo de que el profesor le sugiera que es mejor que pase por su despacho otro día, sobre todo si la irrupción se produce en fin de semana. La razón probable es que SuMMERS esté siguiendo con atención a través del televisor un partido de fútbol americano, especialmente si lo disputa su equipo favorito, el de la Universidad de Oregón.

Sigamos suponiendo que el visitante no conoce nada sobre el juego del fútbol americano, dado que es un deporte que sólo se practica con carácter mayoritario en Norteamérica. Cuando finalmente se reúna con el profesor SuMMERS, es de esperar que le pregunte en qué consiste ese juego que le tenía tan interesado (y que tal vez sea causa de cierta euforia durante su conversación, si es que la Universidad de Oregón venció el encuentro).

Imaginemos que como única respuesta SUMMERs le entrega al visitante una copia del reglamento del fútbol americano. Aceptemos que se trata de una copia fiel y autoritativa, emitida por la agencia o el órgano, cualquiera que éste sea, que rija la reglamentación de ese juego. ¿Sería una respuesta apropiada a la pregunta? ¿Estaría dándole una buena explicación sobre en qué consiste el fútbol americano? Me parece que no, y estoy convencido de que SUMMERS no haría eso, puesto que sería incoherente con la actitud que subyace a su teoría jurídica ${ }^{27}$.

En efecto, entregándole el reglamento al visitante le ofrecería una información apropiada y relevante sobre el fútbol americano, pero extremadamente limitada a la hora conocer y comprender el fenómeno. Después de leer el reglamento, un ignorante del juego podría ser capaz de enumerar cosas tales como el tipo de sanciones disciplinarias

27 Summers utiliza expresamente la analogía del juego (de cualquier juego) para resaltar el alcance limitado de la perspectiva de la regla a la hora de captar, por ejemplo, la forma y el funcionamiento de una institución jurídica (F\&F 100). 
que existen, las causas en las que hay que incurrir para que se impongan, o las condiciones para lograr tantos. Pero a pesar de eso su incomprensión del juego podría ser inmensa. No tendría demasiada idea sobre el aspecto general de la actividad y de su desarrollo, y es plausible pensar que sería totalmente incapaz de identificarla. No sabría nada sobre las disposiciones tácticas, sobre los estilos de juego, sobre las estrategias que se emplean para lograr la victoria, sobre las habilidades que hacen que los jugadores sean más o menos buenos. Tampoco sabría nada sobre los valores atribuidos al juego, o sobre las causas que despiertan afición e incluso admiración. Seguiría sin poder entender de qué manera el juego atrae la atención de personas inteligentes, y por supuesto no podría dar crédito de la expectación que se genera en el país entero durante la disputa de un partido importante. En definitiva, conocería muy poco sobre el fenómeno del fútbol americano aunque supiese de memoria el reglamento del juego y entendiese el contenido de las normas según el sentido ordinario de sus palabras ${ }^{28}$.

Creo que esta analogía capta bien la esencia de la teoría de SuMMERS: algo parecido a lo que le sucede a ese hipotético desconocedor del fútbol americano es lo que nos sucede a todos cuando intentamos conocer y comprender el Derecho a través de las reglas, o sea, utilizando un rule-oriented approach. Ante lo cual cabe preguntarse, ¿sitúa esto a SuMMERS fuera del positivismo? ¿Es su teoría, que se presenta explícitamente por contraste con las de HART y KELSEN, antipositivista?

Si se permite la licencia, creo que más que de teoría antipositivista habría que hablar de apositivista. Es decir, que al autor la cuestión no le preocupa en demasía. Una importante indiciación de ello es que cuando decide afrontar este tema expresamente, lo hace en apenas un párrafo, que además emplea en señalar un punto al que no concede una posición central en su teoría ${ }^{29}$.

Está claro que si entendemos el positivismo en términos de separación metodológica entre ser y deber-ser, la teoría de SuMMERS es incompatible con el mismo ya que combina declaradamente las dimensiones descriptiva y evaluativa (F\&F 88). Por otra parte, si entendemos el positivismo en términos de la tesis de las fuentes sociales del Derecho, la teoría de SuMMERS difícilmente encaja con ella, ya que en la tesis de las fuentes sociales, al menos tal como se formula habitualmente, se presupone la equiparación entre Derecho y normas, mientras que la idea de fondo de SuMMERS es que debemos analizar el Derecho a partir de otros elementos distintos a los normativos. Así, desde la

${ }_{28}$ También utiliza una analogía con el fútbol americano el profesor L. HiERRO, 2002, en su artículo «¿Por qué ser positivista?», DOXA, 25, pp. 265-266. Sin embargo, el propósito allí es distinto del que aquí se pretende. HIERRO utiliza el ejemplo para ilustrar el punto de vista interno, o del participante, de HART: un espectador de un partido de fútbol americano que desconozca por completo las reglas sólo verá regularidades (golpes, carreras, apelotonamientos y reagrupamientos sucesivos...) sin ser capaz de entender nada del juego, ni por tanto del comportamiento de los jugadores, árbitros o aficionados. Es evidente que al conocer la regla el espectador se halla en una mejor posición para entender el fenómeno, y en última instancia poder ser partícipe del mismo. Eso es correcto y no se niega aquí, de la misma manera que SuMMERS no niega que las reglas sean un elemento importante del fenómeno jurídico.

29 SuMmers dice que la teoría de la forma no es positivista porque una ley puesta puede ser tan deficiente desde el punto de vista formal que no merezca ser considerada Derecho (F\&F 34). Esta idea podía haber sido objeto de una amplia discusión y clarificación, de la que sin embargo SUMMERS no se ocupa. Le dedica tan sólo un párrafo en una obra de casi cuatrocientas páginas. Antes, Summers había afirmado en una nota al pie: «Permítaseme añadir que no soy un positivista en ninguno de los sentidos usuales de esa palabra tan abusada» (F\&F 26, n. 75). 
perspectiva de la teoría general de la forma, el clásico debate entre el iusnaturalismo y el positivismo jurídico en torno al origen y la naturaleza de las normas resulta pobre en sí mismo, ya que centra toda la discusión en un único tipo de unidad jurídica funcional (el preceptivo) de entre varias.

Por eso digo que más que antipositivista, a SuMMERs habría que considerarlo apositivista. Su teoría no se ocupa en destacar inconsistencias internas del positivismo (en particular, su respeto por la versión hartiana es notorio), sino que más bien marca sus límites y resalta lo mucho que aquél deja oscurecido, pasando así por encima de debates seculares. Una actitud que me parece coherente con el carácter innovador de su obra.

Es esta, en definitiva, una teoría ambiciosa y de valor filosófico, a pesar de no pretender otra cosa que «ofrecer una nueva manera de mirar lo cotidiano» (F\&F xiii); o precisamente por eso. 